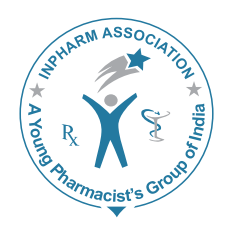

JVP

\title{
Formulation and Evaluation of Solid Lipid Nanoparticles of Ramipril
}

\author{
Ekambaram P, Abdul Hasan Sathali A \\ Department of Pharmaceutics, Madurai Medical College, Madurai, Tamilnadu, India \\ Address for correspondence: Prof. A. Abdul Hasan Sathali; E-mail: abdulhasan@hotmail.com
}

\begin{abstract}
Solid lipid nanoparticles are typically spherical with an average diameter between 1 and $1000 \mathrm{~nm}$. It is an alternative carrier system to tradition colloidal carriers, such as, emulsions, liposomes, and polymeric micro and nanoparticles. Ramipril is an antihypertensive agent used in the treatment of hypertension. Its oral bioavailability is $28 \%$ and it is rapidly excreted through the renal route. This drug has many side effects such as, postural hypotension, hyperkalemia, and angioedema, when given as an immediate dosage form. To overcome the side effects and to increase the bioavailability of ramipril, solid lipid nanoparticles of ramipril are prepared by using lipids (glyceryl monostearate and glyceryl monooleate) with stabilizers (tween 80, poloxamer 188, and span 20). The prepared formulations have been evaluated for entrapment efficiency, drug content, in-vitro drug release, particle size analysis, scanning electron spectroscopy, Fourier transform-infrared studies, and stability. A formulation containing glyceryl monooleate, stabilized with span 20 as surfactant showed prolonged drug release, smaller particle size, and narrow particle size distribution, as compared to other formulations with different surfactants and lipids.
\end{abstract}

Key words: Colloidal carriers, ramipril, lipid matrix, surfactants, entrapment efficiency

\section{INTRODUCTION}

A drug's therapeutic efficacy depends on four fundamental pathways of drug transport and modification within the body, absorption, distribution, metabolism, and elimination. Failure in therapy includes insufficient drug concentration due to poor absorption, rapid metabolism and elimination, poor drug solubility, and high fluctuation of plasma levels

\begin{tabular}{|l|l|}
\hline \multicolumn{2}{|c|}{ Access this article online } \\
\hline Quick Response Code: & \\
\hline & Website: \\
\hline & www.jyoungpharm.in \\
& \\
\hline
\end{tabular}

due to unpredictable bioavailability. A promising strategy to overcome these problems involves the development of a suitable drug colloidal carrier system. Among the colloidal carrier systems solid lipid nanoparticles have many advantages and limited disadvantages as compared to other colloidal carrier systems. Solid lipid nanoparticles (SLN) have gained attention as carriers for the preparation of a wide variety of poorly water soluble drugs due to their biodegradable and biocompatible properties and low toxicity. ${ }^{[1]}$

Ramipril, a potent antihypertensive agent has been used in the treatment of hypertensive disorders. It is a highly lipophillic (log p octanol/water, 3.32) and poorly water soluble drug, with absolute bioavailability of $28-35 \%{ }^{[2]}$ It undergoes significant 'first pass' metabolism and the 
active metabolite of ramipril is ramiprilate. On the basis of the above fact, the aim of this study is to prepare and characterize the ramipril loaded solid lipid nanoparticles, using glyceryl monostearate and glyceryl monooleate as the lipid matrices and tween 80, poloxamer 188, and span 20, as stabilizers, with a view to improve the dissolution rate of Ramipril, which would increase the biological activities. Given that solid lipid nanoparticles, as an alternative colloidal carrier (transport) system, have the ability to improve the solubility/permeability of lipophilic drugs, they may enhance the drug absorption..$^{[3]}$

\section{MATERIALS AND METHODS}

\section{Materials}

Ramipril and Poloxamer 188 were obtained as a gift sample from Madras Pharmaceuticals, Chennai. Glyceryl monostearate [GMS] (CDH Pvt. Ltd., Mumbai), Glyceryl monooleate [GMO] (Otto Chemicals, Mumbai), Polysorbate 80 (Sisco Research Laboratories, Chennai), Sorbitan monolaurate (Loba Chemie, Mumbai), Chloroform and Methanol (Rankem, Chennai), Dialysis Membrane 50 - LA 387 (Himedia, Mumbai) were purchased from the local market. All the reagents used were of analytical grade.

Preparation of ramipril loaded solid lipid nanoparticles

Ramipril loaded SLN were prepared by hot homogenization followed by the ultrasonication method. ${ }^{[4]}$ Ramipril and monoglyceride were dissolved in a mixture of methanol and chloroform (1:1). Organic solvents were completely removed using a rotary flash evaporator. The embedded lipid layer was melted by heating to $5^{\circ} \mathrm{C}$ above the melting point of the lipid. An aqueous phase was prepared by dissolving the stabilizers (tween 80 or poloxamer 188 or span 20) in distilled water (sufficient to produce $30 \mathrm{ml}$ ) and heating to the same temperature of the oil phase. The hot aqueous phase was added to the oil phase and homogenization was performed (at $2500 \mathrm{rpm}$ and $70^{\circ} \mathrm{C}$ ) using a mechanical stirrer for 30 minutes. The coarse oil in water emulsion so obtained was sonicated using probe soincator for 25 minutes. Ramipril loaded SLN was finally obtained by allowing the hot nanoemulsion to cool to room temperature, and was stored at $4^{\circ} \mathrm{C}$ in the refrigerator. The composition of the different formulation has been given in Table 1.

In all SLN formulations the lipid concentration was kept constant (as 6\% w/v).

\begin{tabular}{|c|c|c|c|c|c|}
\hline Formulation & Solid lipid & $\% W / V$ & Surfactant & $\% W / V$ & $\begin{array}{c}\text { Entrapment } \\
\text { efficiency } \\
\% \pm \text { S.D* }\end{array}$ \\
\hline F1 & GMS & 6 & Tween 80 & 1.0 & $78.72 \pm 0.64$ \\
\hline F2 & GMS & 6 & Tween 80 & 1.5 & $80.06 \pm 0.47$ \\
\hline F3 & GMS & 6 & Tween 80 & 2.0 & $81.22 \pm 0.52$ \\
\hline $\mathrm{F} 4$ & GMS & 6 & Poloxamer 188 & 1.0 & $81.97 \pm 0.79$ \\
\hline F5 & GMS & 6 & Poloxamer 188 & 1.5 & $84.13 \pm 0.32$ \\
\hline F6 & GMS & 6 & Poloxamer 188 & 2.0 & $85.70 \pm 0.26$ \\
\hline F7 & GMS & 6 & Span 20 & 1.0 & $72.50 \pm 0.79$ \\
\hline F8 & GMS & 6 & Span 20 & 1.5 & $74.85 \pm 0.10$ \\
\hline F9 & GMS & 6 & Span 20 & 2.0 & $76.64 \pm 0.11$ \\
\hline F10 & GMO & 6 & Tween 80 & 1.0 & $75.24 \pm 0.05$ \\
\hline F11 & GMO & 6 & Tween 80 & 1.5 & $76.62 \pm 0.53$ \\
\hline F12 & GMO & 6 & Tween 80 & 2.0 & $77.59 \pm 0.18$ \\
\hline F13 & GMO & 6 & Poloxamer 188 & 1.0 & $79.82 \pm 0.24$ \\
\hline F14 & GMO & 6 & Poloxamer 188 & 1.5 & $84.48 \pm 0.45$ \\
\hline F15 & GMO & 6 & Poloxamer 188 & 2.0 & $86.40 \pm 0.24$ \\
\hline F16 & GMO & 6 & Span 20 & 1.0 & $74.97 \pm 0.32$ \\
\hline F17 & GMO & 6 & Span 20 & 1.5 & $79.34 \pm 0.17$ \\
\hline F18 & GMO & 6 & Span 20 & 2.0 & $82.71 \pm 0.44$ \\
\hline
\end{tabular}

*S. D - Standard deviation

Evaluation of ramipril-loaded solid lipid nanoparticles

\section{Entrapment efficiency}

The entrapment efficiency of SLN dispersion was determined by the centrifugation method. ${ }^{[5]}$ SLN dispersion (containing an equivalent to $5 \mathrm{mg}$ of drug) was centrifuged at $20000 \mathrm{rpm}$ for one hour in a refrigerated centrifuge to collect the supernatant liquid. The collected liquid was filtered to measure the free drug concentration after suitable dilution with a fresh phosphate buffer saline $\mathrm{pH}$ 7.4. The absorbance was measured at $207 \mathrm{~nm}$ in a UV spectrophotometer ${ }^{[12]}$ to calculate the entrapment efficiency using the following formula:

Entrapment efficiency $=$ Wt. of drug incorporated/ Wt. of drug initially taken $\times 100$

\section{In vitro drug release}

The in vitro release of ramipril from different SLN dispersions was determined using the dialysis bag diffusion technique ${ }^{[6]}$ An accurately weighed amount of Ramipril-loaded SLN dispersions containing the drug equivalent to $2.5 \mathrm{mg}$ was transferred to a dialysis bag and sealed. The sealed bag was then suspended in a beaker containing $250 \mathrm{ml}$ of phosphate buffer saline $\mathrm{pH} 7.4$ and stirred at a constant speed of $50 \mathrm{rpm}$ at $37^{\circ} \mathrm{C} \pm 0.5^{\circ} \mathrm{C}$. Aliquots were withdrawn at predetermined intervals from the receptor compartment up to 12 hours and the same was replaced with fresh buffer. Then the drug content was determined spectrophotometrically by measuring the absorbance at $207 \mathrm{~nm}$ using the respective 
receptor medium as a blank, to calculate the amount of drug released from the nanoparticles.

Particle size analysis/scanning electron microscopy

The mean diameter of the SLNs in the dispersion was determined by photon correlation spectroscopy (PCS) at a fixed angle of $90^{\circ}$, at $25^{\circ} \mathrm{C} \cdot{ }^{[7]}$ Before the measurement, one drop of sample was taken from each selected formulated nanosuspension and diluted in $10 \mathrm{ml}$ of the dispersion medium (distilled water). The surface morphology of SLN was characterized by scanning electron microscopy. ${ }^{[8]}$

\section{Fourier transform-infrared studies}

The interaction between the lipids and drug was identified from the fourier transform-infrared (FT-IR) studies. ${ }^{\left[{ }^{[]}\right.}$

\section{Stability studies}

Stability studies were carried out for the formulations having high entrapment efficiency by storing the formulations at two different temperatures, $4^{\circ} \mathrm{C}$ and $25 \pm$ $2^{\circ} \mathrm{C}^{[10]}$ and the drug content was estimated every 15 days, to find any change in the entrapment efficiency of the SLN.

\section{RESULTS AND DISCUSSION}

\section{Entrapment efficiency}

Entrapment efficiency is an important parameter for characterizing solid lipid nanoparticles. In order to attain optimal encapsulation efficiency, several factors were varied, including the type and concentration of the lipid and surfactant material used. The entrapment efficiency of all the prepared SLN formulations is shown in Table 1. The entrapment efficiency of the SLN dispersions was found to be in the range of 72.50 to $86.40 \%$.

\section{Effect of surfactants on entrapment efficiency}

All SLN formulations prepared with a higher concentration of surfactant $(2.0 \%)$ showed higher entrapment efficiency [F3 - 81.22\%, F6 - 85.70\%, F9 - 76.64\%, F12 - 77.59\%, F15 $-86.40 \%$, and F18 - 82.71\%] irrespective of the type of surfactant. This showed that the increase in the concentration of the surfactant increased the entrapment efficiency. This may be due to the increase in the solubility of the drug in the lipid on increasing the concentration of the surfactant. This result was in agreement with the results obtained by Abdelbary et al. ${ }^{[7]}$ Formulations containing span 20 as surfactants showed lower entrapment efficiency compared to the other surfactants; this could be due to the lower HLB value of Span 20. Hence the entrapment efficiency of various SLNs stabilized with different nonionic surfactants, and decreased in the order of poloxamer $188>$ tween $80>$ span 20 .

\section{In vitro drug release}

The in vitro drug release profile of ramipril from various SLN formulations is shown in [Figure 1]. The in vitro release of ramipril from the SLN dispersion was found to be in the range of 54.90 to $81.40 \%$ at the end of 12 hours.

Influence of surfactants on in vitro drug release

Formulations [F3, F6 and F9] prepared by using GMS as a lipid matrix, with Tween $802.0 \%$, Poloxamer 188 $2.0 \%$, and Span $202.0 \%$ as stabilizers showed a higher drug release from Ramipril-loaded SLN [79.46, 81.21, and $64.80 \%$, respectively]. This showed that the increase in the concentration of the surfactant there was an increase in the drug release from the SLN. However, this difference could not be attributed to the formulations [F12, F15 and F18] prepared by using GMO as lipid matrix with tween 80 $2.0 \%$, poloxamer $1882.0 \%$, and span $202.0 \%$, respectively. These formulations showed a decrease in the drug release on the increase of the concentration of surfactant, which could be due to the lower melting point of the GMO than the GMS. The results indicated that formulation [F6 - GMS + Poloxamer 188 2.0\%, (81.40\%)] exhibited a higher drug release and formulation [F18 - GMO + Span $202.0 \%$, $(54.90 \%)]$ showed a higher retarded drug release among the three surfactants studied. This could be due to the lower HLB value of Span 20 (8.6) than the other surfactants used as stabilizers. Thus the order of percentage of drug release was Poloxamer $188>$ Tween $80>$ Span 20 on the basis of the stabilizer.

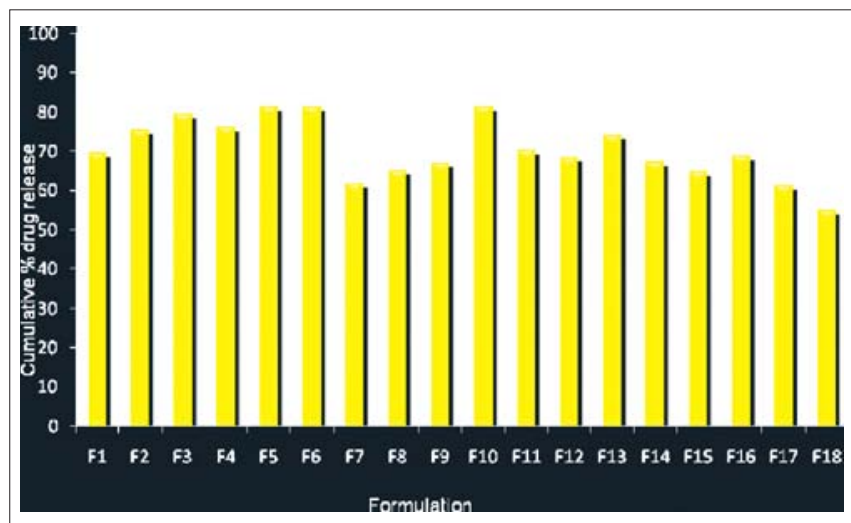

Figure 1: Comparison of in vitro drug release profile of ramipril from various solid lipid nanoparticle formulations at the end of 12 hours 


\section{Influence of lipids on in vitro drug release}

Formulations containing GMS as a lipid matrix exhibited higher drug release than the formulations containing GMO as the lipid matrix, which showed a more sustained release. GMO produced less ordered crystals than GMS, leading to lower drug expulsion from the imperfect lattice, contributing to the prolonged release of the lipophilic drug. Moreover, GMO was a lipid with a lower melting point when compared to GMS. Zur Muhlen et al. ${ }^{[1]]}$ reported that a lower melting point could produce a controlled release from SLN. The order of percentage of drug release was Glyceryl monostearate $>$ Glyceryl monooleate on the basis of the lipid matrix. Thus, it could be well concluded that the amount of drug released was much slower and controlled from the SLN dispersions prepared by using GMO as the lipid matrix, with Span 20 as the surfactant, than that from the ramipril-pure drug solution [Figure 2].

Particle size analysis

Figure 3 shows the effect of the lipid and surfactant concentration on the particle size distribution of ramiprilloaded SLN prepared by using GMS and GMO with poloxamer $1882.0 \%$, tween $802.0 \%$, and span $202.0 \%$. The particle size ranged from $104 \mathrm{~nm}-334 \mathrm{~nm}$. There was a significant difference in the size of the particles, with change in the lipids.

\section{Effect of lipids on the particle size of ramipril SLN}

Ramipril-loaded solid lipid nanoparticles, prepared by using GMS as the lipid matrix, resulted in larger particle size compared to SLN prepared by using GMO as the lipid matrix, with all type of surfactants studied. This phenomenon could be attributed to the melting point of the lipid, GMS has a higher melting point than GMO, which results in a slower lipid crystallization from the hot homogenized condition resulting in an increase in the particle size. The particle size of various SLNs prepared with different lipids was in the order of GMS > GMO.

\section{Effect of surfactants on the particle size of ramipril SLN}

SLN dispersion prepared using poloxamer $1882.0 \%$ as stabilizer [F6 and F15] showed lower particle size than the other surfactants irrespective of the lipids studied. This result could be explained due to the higher molecular weight of poloxamer 188 and higher HLB value of poloxamer 188 when compared to tween 80 and span 20. ${ }^{[12]}$ The particle size of various SLN, stabilized with different surfactants, increased in the order of Poloxamer $188>$ Tween $80>$ Span 20.

\section{Scanning electron microscopy}

SEM studies showed that the ramipril-loaded solid lipid nanoparticles had a spherical shape with a smooth surface as shown in Figure 4.

\section{FT-IR studies}

Infrared studies were carried out to confirm the compatibility between the lipid, drug, and selected SLN formulation. From the spectra it was observed that there was no major shifting, as well as, no loss of functional peaks between the spectra of

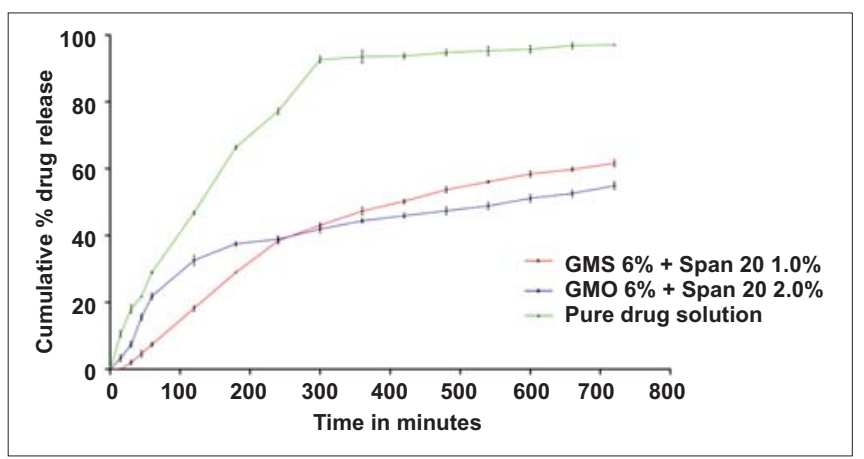

Figure 2: Comparison of in vitro drug release of SLN prepared by using GMS 6\%, GMO 6\% and pure drug solution

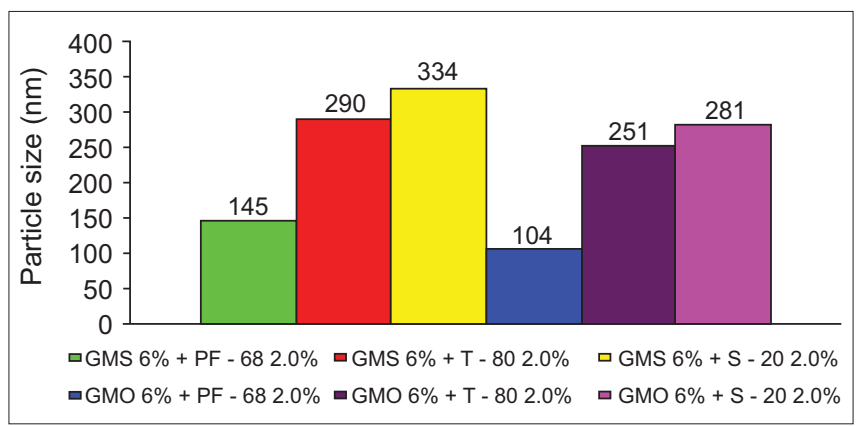

Figure 3: Comparison of particle size of SLN of GMS 6\% and GMO $6 \%$ (PF - 68 2.0\%, T - $802.0 \%$, and S - $202.0 \%$ )

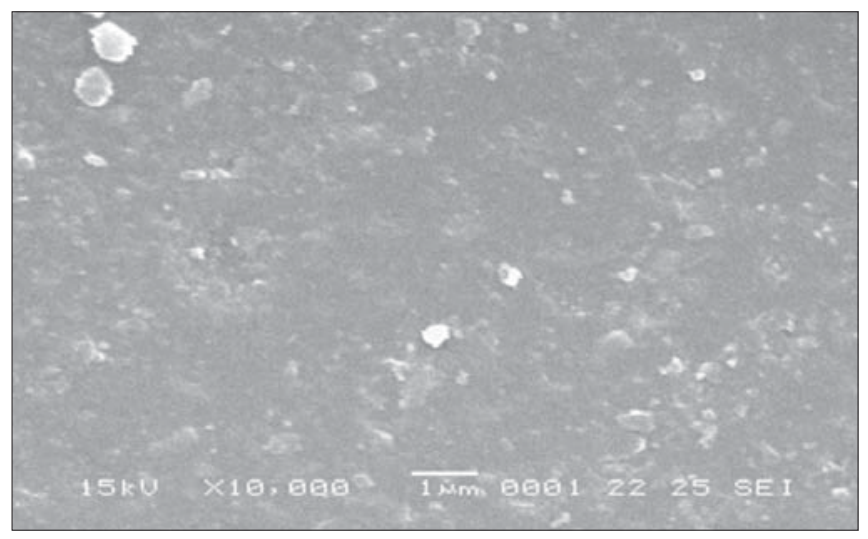

Figure 4: F15 - GMO 6\% + Poloxamer 188 2.0.\% 


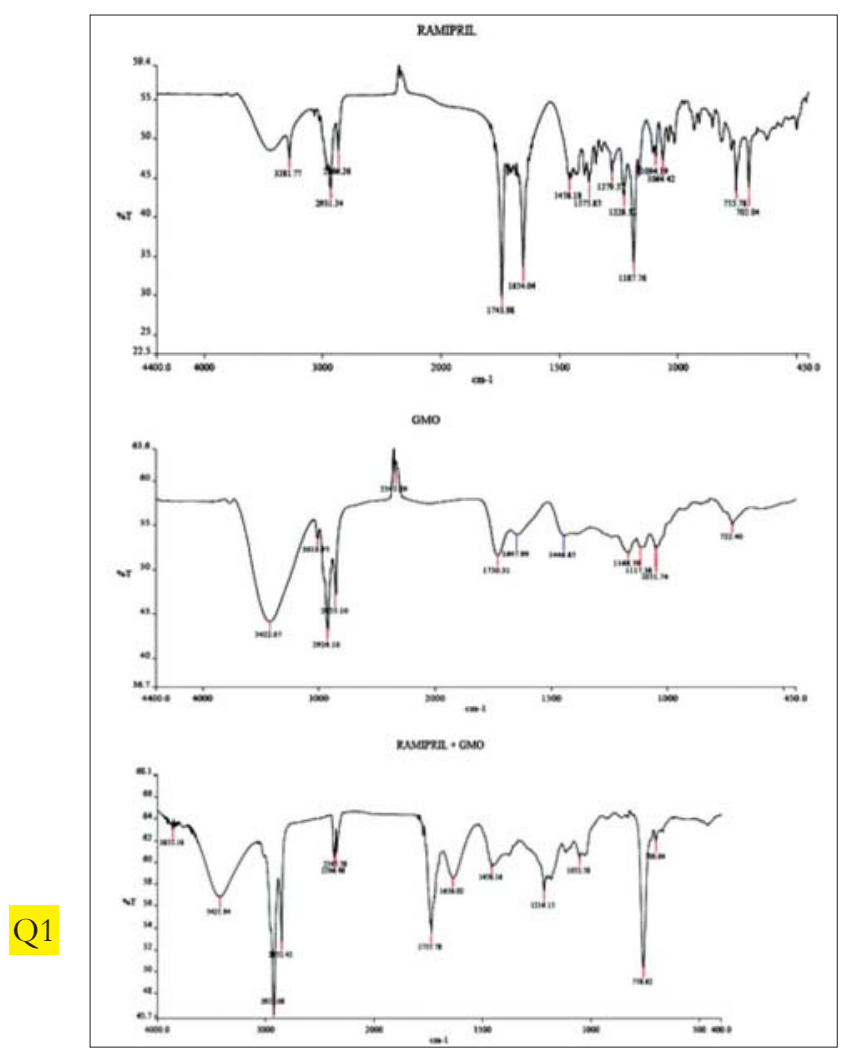

Figure 5: Comparison of I.R spectrum of pure drug, GMO and pure drug + GMO

the drug, lipid, and drug-loaded SLN. [1743.98 $\mathrm{cm}^{-1}, 4654.04$ $\mathrm{cm}^{-1}, 1458.18 \mathrm{~cm}^{-1}$, and $\left.2931.34 \mathrm{~cm}^{-1}\right]$. This indicated no interaction between the drug and lipid [Figure 5].

\section{Stability studies}

The results showed that the entrapment efficiency of the SLN dispersion stored in $4^{\circ} \mathrm{C}$ was more compared to the SLN dispersion stored in $25^{\circ} \mathrm{C} \pm 2^{\circ} \mathrm{C}$. This may be due to more drug expulsion from the lipid matrices at higher temperature [Table 2].

\section{CONCLUSION}

It was observed that the hot homogenization and ultrasound dispersion method was a useful method for the successful incorporation of the poor watersoluble drug ramipril with high entrapment efficiency. Furthermore, it could be presumed that if the nanometer range particles were obtained, the bioavailability might be increased. Hence, we can conclude that solid lipid nanoparticles provide controlled release of the drug
Table 2: Stability studies for solid lipid nanoparticles (Percentage of entrapment efficiency at refrigerated temperature and $25^{\circ} \pm 2^{\circ} \mathrm{C}$ )

\begin{tabular}{|c|c|c|c|c|c|}
\hline \multirow[t]{2}{*}{ Formulation } & \multirow{2}{*}{$\begin{array}{c}\text { Immediately after } \\
\text { preparation }(\%)\end{array}$} & \multicolumn{2}{|c|}{$15^{\text {th }}$ day $(\%)$} & \multicolumn{2}{|c|}{$30^{\text {th }}$ day $(\%)$} \\
\hline & & $4^{\circ} \mathrm{C}$ & $25^{\circ} \pm 2^{\circ} \mathrm{C}$ & $4^{\circ} \mathrm{C}$ & $25^{\circ} \pm 2^{\circ} \mathrm{C}$ \\
\hline F3 & 81.22 & 81.06 & 78.16 & 80.00 & 75.31 \\
\hline F6 & 85.70 & 85.06 & 82.90 & 83.94 & 77.91 \\
\hline F9 & 76.64 & 76.32 & 73.56 & 74.47 & 69.19 \\
\hline F12 & 77.59 & 77.02 & 74.02 & 75.81 & 70.01 \\
\hline F15 & 86.40 & 86.00 & 85.26 & 83.16 & 81.38 \\
\hline F18 & 82.71 & 82.15 & 81.86 & 81.57 & 77.69 \\
\hline
\end{tabular}

and these systems are used as drug carriers for lipophilic drugs, to enhance the bioavailability of poorly watersoluble drugs through nanoparticles, as a drug delivery system.

\section{REFERENCES}

1. Delie F, Blanco-Príeto MJ. Polymeric particulates to improve oral bioavailability of peptide drugs. Molecules 2005;10:65-80.

2. AHFS; Drug Information, American society of Health-system Pharmacists, Inc. 7272 Wisconsin Avenue, Bethseda, MD 20814,2004:1869-75.

3. Mukherjee S, Ray S, Thakur RS. Solid lipid nanoparticles: A modern formulation approach in drug delivery system. Indian J Pharm. Sci 2009;71:349-58.

4. Müller RH, Mäder K, Gohla S. Solid lipid nanoparticles (SLN) for controlled drug delivery - A review of the state of the art. Eur J Pharm Biopharm 2000;50:161-77.

5. Luo Y, Chen D, Ren L, Zhao X, Qin J. Solid lipid nanoparticles for enhancing vinpocetine's oral bioavailability. J Control Release 2006;114:53-9.

6. Huang G, Zhang N, Bi X, Dou M. Solid lipid nanoparticles of temozolomide: Potential reduction of cardial and nephric toxicity. Int J Pharm 2008;355:314-20.

7. Abdelbary G, Fahmy RH. Diazepam - loaded solid lipid nanoparticles: Design and characterization. AAPS PharmSciTech 2009;10:211-9.

8. Heinzelmann ME, Wiesendanger R. Scanning tunneling microscopy II surface science. Newyork: Springer verlogg; 1992. P. 99-149.

9. Bhalekar MR, Pokharkar V, Madgulkar A, Patil N, Patil N. Preparation and evaluation of miconazole nitrate-loaded dolid lipid nanoparticles for topical delivery. AAPSPharmSciTech 2009;10:289-96.

10. Suresh G, Manjunath K, Venkateswarlu V, SatyanarayanaV. Preparation, characterization, in-vitro and in-vivo evaluation of lovastatin solid lipid nanoparticles. AAPS PharmSciTech 2007 Mar 23;8:24.

11. Zur Mühlen A, Schwarz C, Mehnert W. Solid lipid nanoparticles (SLN) for controlled drug delivery - Drug release and mechanism. Eur J Pharm Biopharm 1998;45:149-55.

12. Jawahar N, Eaggapanath T, Nagaswamy V, Samantha JS. Preparation and characterization of PLGA - Nanoparticles containing a hypertensive agent. Int J Pharm Tech Res 2009;2:390-3.

How to cite this article: Ekambaram P, Sathali AA. Formulation and evaluation of solid lipid nanoparticles of ramipril. J Young Pharmacists 2011;3:216-20.

Source of Support: Nil, Conflict of Interest: None declared. 